\title{
A full new enthusiastic team
}

\author{
Loreto Carmona
}

Received: 28 February 2013/Accepted: 8 March 2013/Published online: 15 March 2013

(c) Springer-Verlag Berlin Heidelberg 2013

Science is about systematic procedures, about reproducibility, sharing methods, results and limitations, and about submitting our studies to the eye of others for critic. Science is not about showing how good we are, but about showing how things are. Science is hard and competitive; avoiding biases; knowing that you will never hold the truth and that somebody will come and prove you wrong. But science is what makes us free and better.

We need this. We have to stand firm in front of those with stronger voices or those in better position to reach and to manipulate the public. Scientific journals are the voice and reason of science and we need to show that everything we do complies with all ethical standards today. Strict publication policies and rules to perform research do not look kind to many, but they guide us on how to continually improve with the highest standards in place. We are "playing" with health, ladies and gentleman, so we have to make sure we know how to use data, how to report it and how to incorporate it to our daily practice. We therefore need you; and we need you at your best.

At some point in time-crazy world this is-somebody must have told us to rush and to forget about enjoying what we do. We rush to study, we rush to work, we rush to collect data-almost forgetting to write a protocol firstwe rush to submit our data to a thousand congresses, to get them published. That is not the way to go. Why race to publication whatever it takes? What are we doing with science? What are we doing with life? Something I have

\footnotetext{
L. Carmona $(\bowtie)$

Institute for Musculoskeletal Health, Madrid, Spain

e-mail: loreto.carmona@inmusc.eu

L. Carmona

Universidad Camilo José Cela, Madrid, Spain
}

learnt in years of writing, reviewing and editing papers, abstracts and grants, is that it is possible to tell who has enjoyed the process, because it is always evident in the writing. Only through enjoying science do we produce the best research. We have to train our younger counterparts, that is, science is also about education and that is about taking care of every piece of research as if it were their baby, a cake or a jewel. This way we will all enjoy reading science again.

Professor Lemmel founded this Journal in 1980 and has been the Editor-in-Chief for over 30 years. It has been his baby whom we have seen grow into the successful adult it is now. Professor Lemmel made Rheumatology International the home to many scientists from all over the world, a truly international hub, where people, scientists from all the continents, felt safe. We must thank him for his devoted work and for the creation of this responsive community.

And now here we are. We are not young; we are not old; we are not famous but we are not unknown; we work hard in our careers and we enjoy what we do. If any, the minimum common denominator of the new Editorship of the Journal is enthusiasm and in the hope that science will be the only branch we will be able to hold to in this storm that it is the world today. We are therefore committed to increasing the scientific value of the Journal and its future.

However, at the same time, we recognize our limitations. There are higher impact journals than ours around but we also know that for some unclear reason, there are topics that are difficult to publish in rheumatology or in other specialized areas when written by rheumatologists: matters such as educational interventions or public health issues related to rheumatic and musculoskeletal diseases, epidemiology, non-pharmacological interventions, special populations, cross-cultural validations, etc. These are some of the new frontiers we want to cross in this Journal, to 
maintain the name it holds as a truly "International" Journal. Unfortunately, this also means that we are closing, at least temporarily, some doors that remain open in other journals, including other Springer journals, such as case reports or basic science, which we currently consider we are not prepared to deal with at the same level other journals do. 